\title{
Structured illumination microscopy using digital micro-mirror device and coherent light source
}

Cite as: Appl. Phys. Lett. 116, 233702 (2020); https://doi.org/10.1063/5.0008264

Submitted: 21 March 2020. Accepted: 15 May 2020. Published Online: 09 June 2020

Meiqi Li (iD), Yaning Li (D), Wenhui Liu (D), Amit Lal ${ }^{(\mathbb{D})}$, Shan Jiang (D), Dayong Jin (D), Houpu Yang (D), Shu Wang (D), Karl Zhanghao $(\mathbb{D})$, and Peng Xi $(\mathbb{D})$

\section{COLLECTIONS}

F This paper was selected as Featured

SCl This paper was selected as Scilight

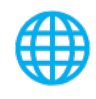

\section{ARTICLES YOU MAY BE INTERESTED IN}

Digital mirror device combined with microscopy technique illuminates biological mechanisms Scilight 2020, 241103 (2020); https://doi.org/10.1063/10.0001456

Study of detailed balance between excitons and free carriers in diamond using broadband terahertz time-domain spectroscopy

Applied Physics Letters 116, 231102 (2020); https://doi.org/10.1063/5.0006993

Time-resolved emission and scattering imaging of plume dynamics and nanoparticle ejection in femtosecond laser ablation of silver thin films

Applied Physics Letters 116, 234105 (2020); https://doi.org/10.1063/5.0009227

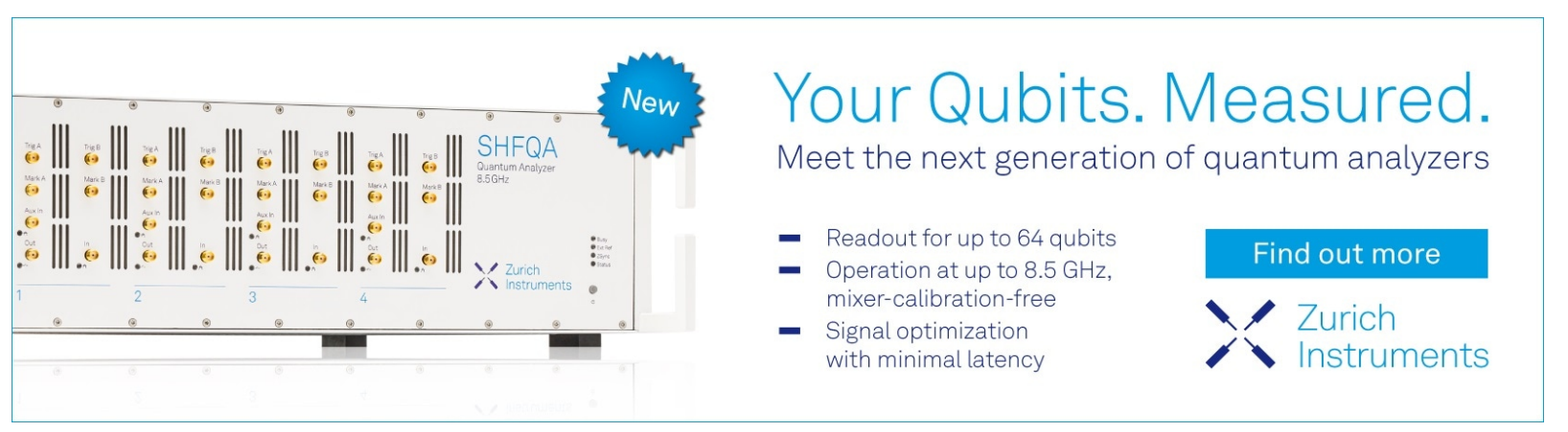




\title{
Structured illumination microscopy using digital micro-mirror device and coherent light source $\odot$ ()
}

Cite as: Appl. Phys. Lett. 116, 233702 (2020); doi: 10.1063/5.0008264

Submitted: 21 March 2020 - Accepted: 15 May 2020 .

Published Online: 9 June 2020

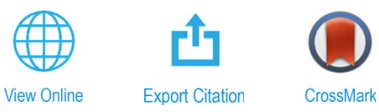

Meiqi Li, (iD Yaning Li,' (iD Wenhui Liu, ${ }^{1}$ (iD Amit Lal, ${ }^{3}$ iD Shan Jiang, (iD Dayong Jin, ${ }^{3}$ (iD Houpu Yang, Shu Wang, ${ }^{4}$ (D) Karl Zhanghao, ${ }^{1,3, a)}$ (iD and Peng Xi ${ }^{1,3, a)}$ (iD)

\author{
AFFILIATIONS \\ ${ }^{7}$ Department of Biomedical Engineering, College of Engineering, Peking University, Beijing 100871, China \\ ${ }^{2}$ Department of Automation, Tsinghua University, Beijing 100084, China \\ ${ }^{3}$ Department of Biomedical Engineering, College of Engineering, Southern University of Science and Technology, \\ Shenzhen 518055, Guangdong, China \\ ${ }^{4}$ Breast Center, Peking University People's Hospital, Beijing 100044, China
}

${ }^{a)}$ Authors to whom correspondence should be addressed: karl.hao.zhang@gmail.com and xipeng@pku.edu.cn

\begin{abstract}
Structured illumination microscopy (SIM) achieves doubled spatial resolution through exciting the specimen with high-contrast, highfrequency sinusoidal patterns. Such an illumination pattern can be generated by laser interference or incoherent structured patterns. Opto-electronic devices, such as a Spatial Light Modulator (SLM) or a Digital Micro-mirror Device (DMD), can provide rapid switch of illumination patterns for SIM. Although the DMD is much more cost-effective than the SLM, it was previously restricted in association with incoherent light sources, as its diffractive orders are related to the incident angle and the wavelength of coherent incidence. To extend its application with coherent illumination, here, we model the DMD as a blazed grating and simulate the effect with DMD pattern changes in the SIM. With careful analysis of the illumination contrast along different angles and phases, we report a fast, highresolution, and cost-efficient SIM with DMD modulation. Our home-built laser interference-based DMD-SIM (LiDMD-SIM) reveals the nuclear pore complex and microtubule in mammalian cells with doubled spatial resolution. We further proposed the multi-color LiDMD-SIM concept by jointly employing the DMD ON/OFF states with different incident angles for different wavelengths, with high contrast and maximum resolution enhancement.
\end{abstract}

Published under license by AIP Publishing. https://doi.org/10.1063/5.0008264

Biologists favor Structured Illumination Microscopy (SIM) for its high resolution, fast imaging speed, and low photo-toxicity. ${ }^{1-4}$ Unlike other super-resolution techniques ${ }^{5}$ such as Stimulated Emission Depletion (STED $)^{6-8}$ and Single Molecule Localization Microscopy (SMLM), ${ }^{9-11}$ SIM is compatible with samples prepared for conventional fluorescence microscopy without requiring special sample labeling effort. ${ }^{12,13}$ Recent studies successfully resolve the ultrastructure of cellular organelles and capture their dynamics using SIM. ${ }^{14,15}$ Furthermore, a recent work exploits the polarization nature of SIM and achieves super-resolution dipole imaging on existing SIM systems. ${ }^{16}$

The key to obtaining super-resolution in SIM is to excite the specimen with high-contrast, fine illumination patterns, which brings high-frequency information into the low-frequency region in reciprocal space. In early studies, mechanical rotation and translation of grating switches between different patterns, which is quite slow and limits the imaging speed. Afterward, an opto-electro device, such as a liquid crystal Spatial Light Modulator (SLM), ${ }^{14-17}$ is adopted into the SIM system to achieve fast pattern switching. The imaging speed achieved $\sim 100$ reconstructed frames per second and captured the fast dynamics of Endoplasmic Reticulum (ER), ${ }^{15}$ mitochondria, ${ }^{2}$ and their interactions. ${ }^{14}$

A Digital Micro-mirror Device (DMD) is one of the most common opto-electro devices in the industry. It provides higher pattern refresh frequency and is offered at a much lower price compared to the SLM, which has been used in Total Internal Reflection Fluorescence (TIRF) microscopy ${ }^{18}$ and optical section microscopy. ${ }^{19}$ Nevertheless, the use of the DMD is seldomly exploited in SIM. Dan et al. ${ }^{20,21}$ reported the first LED-DMD-SIM system that projects the DMD pattern illuminated by an incoherent LED light source. However, LED-DMD-SIM cannot achieve a doubled spatial resolution in experiment, as the contrast of LED pattern is compromised by the system OTF cutoff (Fig. S1, supplementary material). 
In this work, we demonstrate that the incident laser must meet the blaze criteria of the DMD to obtain a high-contrast illumination pattern. To simplify the problem, we only consider 2D-SIM here, while it is also true for 3D-SIM. We start with the simulation of the \pm 1 order light intensities generated by DMD diffraction, to assist the design of the SIM system with a high contrast pattern. Furthermore, we built a laser interference-based DMD-SIM (LiDMD-SIM) system using a single-wavelength laser source. The measured diffractive pattern from the DMD is in accordance with the simulation results. At the end, our LiDMD-SIM system imaged both standard specimens of fluorescent beads and biological specimens such as nuclear pore complexes (NPCs) and microtubules with a half price and the same frame rate of the SLM-SIM system. Fourier Ring Correlation (FRC) analysis demonstrated a doubled spatial resolution in our system.

An intuitive realization of the LiDMD-SIM system is to replace the SLM with a DMD in the laser-interference-based setup. ${ }^{22,23}$ The obstacle preventing such implementation is the nature of the DMD as a blazed grating. The grating is blazed only when the blaze angle, spacing, wavelength, and incident angle cooperate with each other. The blaze angle and spacing are determined by the DMD so that LiDMDSIM should choose the incident angle and wavelength with a special caution.

DMD is a kind of spatial light modulator consisting of a micromirror array on a CMOS memory unit. ${ }^{24}$ Each micro-mirror tilted with a given angle $\theta$ along the rotation axis, which is along the diagonal of the square lattice mirrors, just as Figs. 1(a) and 1(b) show. The deflection of each micro-mirror can be controlled separately by loading different voltage signals to each memory unit. Then, the deviation angle of the exit light is changed to realize the modulation of the

(a)
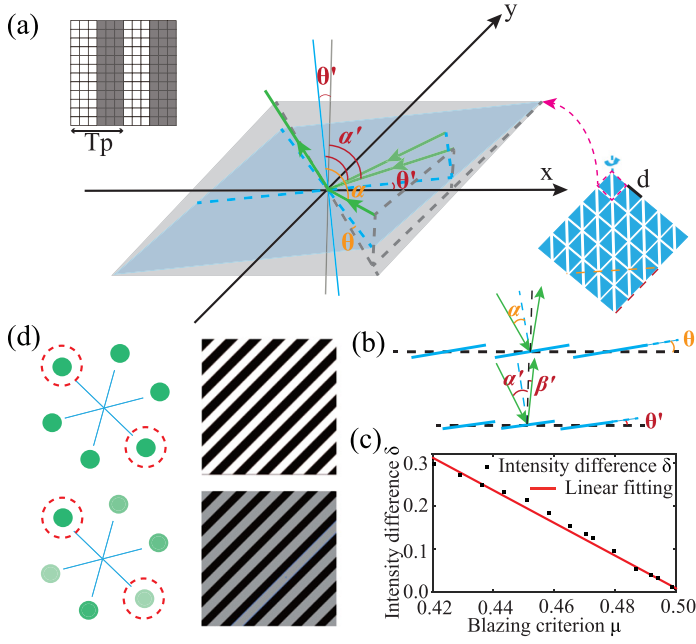

FIG. 1. The theory and simulation results of the diffractive intensity distribution of DMD. (a) The basic structure of the DMD and the pattern loaded on the DMD. The pattern period Tp denotes the periodicity of the grating. $\theta$ along the diagonal of the mirrors can be projected along the axis of the side length of the square lattice mirrors in the flat state as $\theta^{\prime}$. Incident angle $\alpha$ has the same projection relationship. (b) The illustration of the propagation of light on a single micro-mirror. (c) The relationship between the intensity difference and the blaze criterion. (d) The consistent intensity distribution of \pm 1 orders diffractive light produces a high contrast interference pattern (left), while differential light intensity produces a low contrast pattern (right). incident beam. The basic structure of the DMD is shown in Fig. 1(a). Each micro-mirror is controlled in the binary form corresponding to three states: ON state, OFF state, and flat state. When the micromirror is in the $\mathrm{ON}$ state, the incident light will be deflected into the subsequent light path; when the micro-mirror is in the OFF state, the incident light is deflected away from the subsequent light path; the micro-mirror is flat only when the DMD is powered off.

To generate a high-contrast illumination pattern, the two interference beams should have identical intensity, as Fig. 1(d) shows. The diffraction effect of the DMD is similar in the $\mathrm{X}$ and $\mathrm{Y}$ directions, and so we can consider a $1 \mathrm{D}$ problem here. First, we consider that all mirrors are in the ON state. For a tilt angle $\theta$ in the direction of the diagonal of the mirrors [shown in Fig. 1(a)], we can project $\theta$ along the axis of the side length of the square lattice mirrors in the flat state and get $\theta^{\prime}=\arctan (\tan \theta / \sqrt{2})$. The same projection of incident angle $\alpha$ [shown in Figs. 1(a) and 1(b)] is $\alpha^{\prime}=\arctan (\tan \alpha / \sqrt{2})$. Here, we want to emphasize that $\alpha$ and $\alpha^{\prime}$ are starting from different normal axes. $\alpha$ is the angle to the normal axis of the mirror plane, while $\alpha^{\prime}$ is to the normal axis of the DMD plane. To obtain high-efficiency diffraction and consistent intensity of \pm 1 order light, the equivalent incident angle $\alpha^{\prime}$ and reflection angle $\beta^{\prime}$ have to satisfy the grating equation

$$
\sin \left(\alpha^{\prime}\right)-\sin \left(\beta^{\prime}\right)=\frac{\mathrm{m} \lambda}{d},
$$

with $\beta^{\prime}=\alpha^{\prime}-2 \theta^{\prime}$.

$\lambda$ denotes the wavelength of the incident light, and $d$ indicates the pixel pitch of the DMD, which is $7.56 \mu \mathrm{m}$ in our experiment. We can calculate the value $m$ to quantify the blaze condition. The blazing condition is satisfied when $m$ is close to an integer. When $m$ is an integer plus 0.5 , it will be the farthest deviation from the blaze condition. To better qualify the blaze condition, we defined the blaze criterion $\mu$ as $^{25}$

$$
\mu=|\bmod (\mathrm{m}, 1)-0.5|=\left|\bmod \left(\frac{d}{\lambda}\left(\sin \left(\alpha^{\prime}\right)+\sin \left(\beta^{\prime}\right)\right), 1\right)-0.5\right| .
$$

The symbol $|\mathrm{x}|$ means the absolute value of $\mathrm{x}$. If $\mu$ is close to 0.5 , it is at the blaze condition. When $\mu$ is close to zero, it is far away from the blaze condition. Then, the pattern with period Tp is loaded on the DMD [shown in Fig. 1(a)] to produce \pm 1 order light beams with uniform intensity. The two beams diffracted by the DMD have identical intensity only when the blaze criteria are met. The intensity difference between the \pm 1 order beams will influence the contrast of the interference fringes. The relationship between the blaze criterion $\mu$ and the normalized intensity difference $\delta$ is shown in Fig. 1(c). The contrast decreases when the intensity of the two beams differs from one another. The light intensity difference within $30 \%$, which is acceptable for the experiment, corresponds to the $\mu$ value surpassing 0.42 .

For a given DMD with a fixed pixel pitch $d$ and blaze angle $\theta$, the relationship between the wavelength and the incident angle is certain under the blaze condition. Therefore, we need to find an appropriate incident angle at a specific wavelength of excitation so that two consistent interference beams can be generated. Based on this, we choose incident wavelengths of $473 \mathrm{~nm}$ and $561 \mathrm{~nm}$, which are standard excitation wavelengths of fluorophores. With the simulation results, we 
(a)

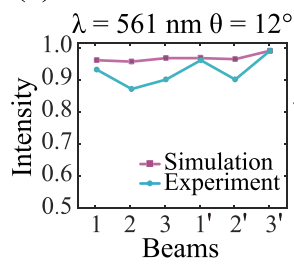

(b)

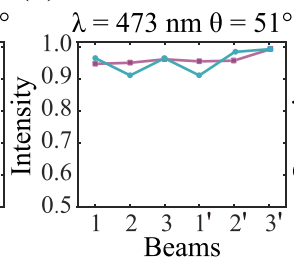

(c)

(d)

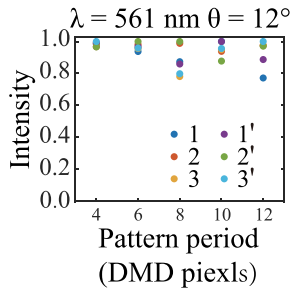

(e)

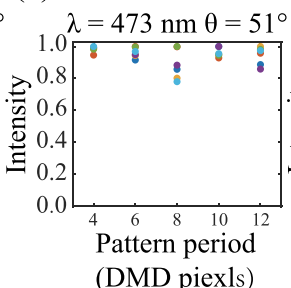

(f)

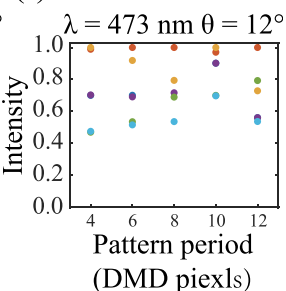

FIG. 2. Experimental verification of DMD diffraction. The intensity distribution of six diffraction beams when the incident angle is $12^{\circ}$ with $561 \mathrm{~nm}$ laser illumination in (a), the incident angle is $51^{\circ}$ with $473 \mathrm{~nm}$ laser illumination in (b), and the incident angle is $12^{\circ}$ with $473 \mathrm{~nm}$ laser illumination in (c). (d)-(f) The relationship between the pattern period and intensity fluctuation under different simulation conditions corresponding to (a), (b), and (c).

figure out the incident angle of $51^{\circ}$ satisfies the blaze condition for $473 \mathrm{~nm}$ excitation and $12^{\circ}$ for $561 \mathrm{~nm}$ excitation, as shown in Figs. 2(a)-2(c).

To verify the simulation results, we build up a LiDMD-SIM imaging system. The schematic of the system is shown in Fig. 3. The expanded laser beam illuminated on the DMD at an incident angle $\alpha$ and is diffracted by the DMD (DLP LightCrafter 6500, Texas Instruments). Then, the generated diffraction orders enter len1. A mask that is especially designed acts as a spatial filter to block the zeroorder beam and keeps the passage at \pm 1 diffraction orders. The distance between the two \pm 1 hole is $4.4 \mathrm{~mm}$, and the size of the hole is $1 \mathrm{~mm}$ in our experiment. The illuminating beam finally comes to the

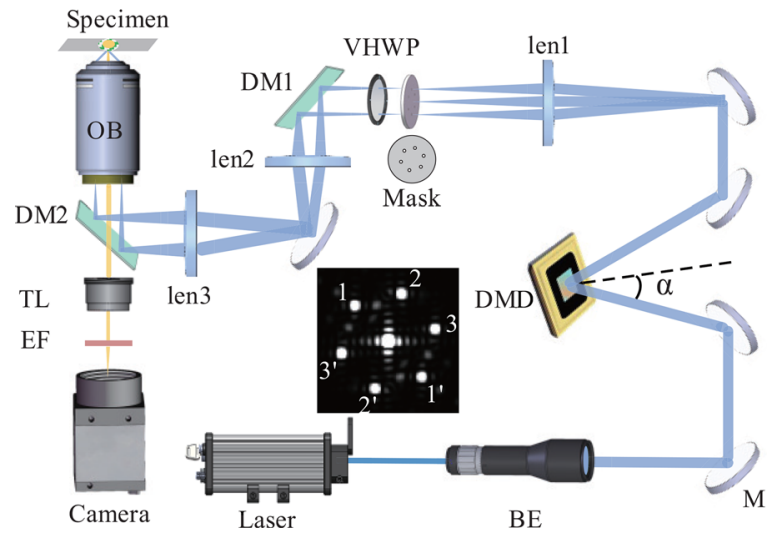

FIG. 3. The system setup of the LiDMD-SIM. BE, Beam Expander; M, Mirror; DMD, Digital Micro-mirror Device; VHWP, Vortex Half-Wave Plate; OB, Objective; DM, Dichroic Mirror; TL, Tube Lens; and EF, Emission Filter. The inset shows the six diffracted beams produced by DMD diffraction. microscope objective through one couple of relay lens (len2 and len3) and interference at the objective focal plane. The Vortex Half-Wave Plate (VHWP) (WPV10L-532, Thorlabs) will change and control the polarization of \pm 1 order beams to make sure that two diffraction beams have identical polarization, which can be displaced by an azimuthal HWP. ${ }^{2,13,14}$ The VHWP avoids the complex manufactured compared to the azimuthal HWP, but it is currently limited to singlecolor application. Three angles and three phases of illumination patterns were realized through the control of each pixel ON-OFF state on the DMD, which is designed in the same way as the SLM. ${ }^{26,27}$ Thus, there are a total of 9 different illumination patterns. The inset image illustrates the \pm 1 order diffracted beams produced by DMD diffraction of three angles. Dichroic mirror is a thin-film interference coating component that will cause the difference polarization effect of the p-plane and the s-plane. So an identical dichroic mirror DM1 is placed such that the s- and p-axes are switched with DM2 to compensate for polarization distortion caused by DM2. ${ }^{16}$ Finally, the fluorescence signal is collected by the objective lens (Nikon, CFI SR Apochromat TIRF 100× oil, NA 1.49) and captured using an sCMOS (Dhyana 400BSI, TUCSEN) camera through a tube lens. The trigger signal sent from the DMD is used for the camera external triggering to ensure the synchronization of acquisition and control.

To ensure that the interference fringes formed by two diffraction beams have high contrast, the two diffraction beams should have the same polarization with equal intensity. The polarization direction of two diffraction beams has been controlled by a VHWP and DM1 to ensure the identical polarization direction. The consistent intensity of the two beams is relevant to the blaze condition of the DMD. We load diffraction grating patterns with a difference of $120^{\circ}$ in three directions into the DMD to generate interference fringes. Then, we measured the intensity of six diffraction beams after the spatial mask (shown in Fig. 3 marked as 1 and $1^{\prime}, 2$ and $2^{\prime}$, and 3 and $3^{\prime}$ ). A comparison of experiment and simulation with normalized diffraction beam intensity in three given conditions is shown in Figs. 2(a)-2(c). When the blaze criterion value is close to 0.5 , the two interference beams will have consistent intensity, such as $\lambda=561 \mathrm{~nm}, \theta=12^{\circ}$ and $\lambda=473 \mathrm{~nm}, \theta=51^{\circ}$. Otherwise, the intensity of the six beams will fluctuate greatly [Fig. 2(c)]. The experimental results are in good agreement with the simulation calculation. We further simulate the relationship between the intensity fluctuation of six beams and the pattern period $\mathrm{Tp}$ we loaded on the DMD. The results show that the intensity coincides well at a specific pattern period under certain conditions. Compared to the incident angle and wavelength, the intensity fluctuation is relatively small when the pattern period changed [shown in Figs. 2(d)-2(f)].

Based on the simulation and experimental analysis, we chose the laser with a central wavelength of $561 \mathrm{~nm}$ (CNI, MGL-FN-561 nm$200 \mathrm{~mW}$ ) as the excitation light source in our LiDMD-SIM imaging system. The incident angle of the excitation beam to the DMD was set to $12^{\circ}$, which is the condition shown in Fig. 2(a). The pattern period Tp is 12 pixels of the DMD in the experiment. We use $100 \mathrm{~nm}$ fluorescent beads with the center emission wavelength of $610 \mathrm{~nm}$ to test the resolution of the LiDMD-SIM system. Since the diameter of the fluorescent beads is smaller than the Abbe diffraction limit, the size of the beads can be neglected during the resolution analysis. To evaluate the resolution better, we use $\mathrm{FRC}^{28}$ analysis to calculate the resolution of the Wide-Field (WF) and SIM results, as shown in Fig. 4(c). FRC is 

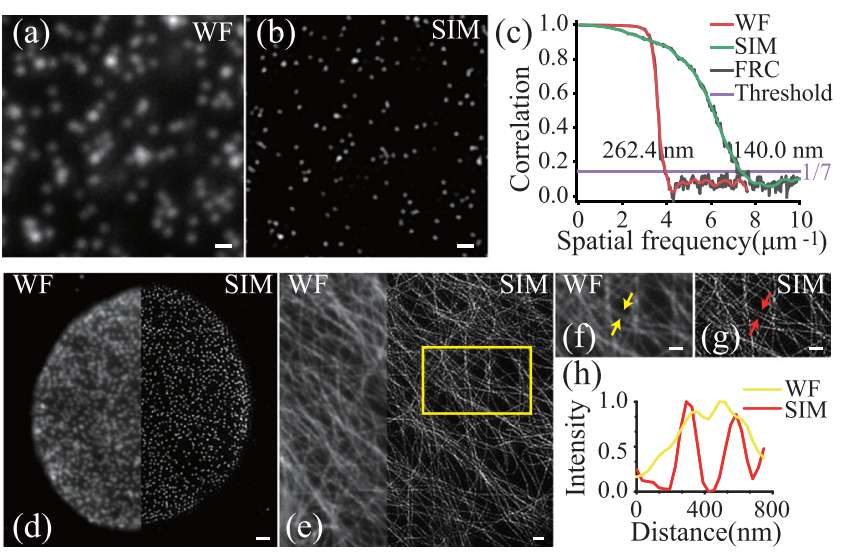

FIG. 4. LiDMD-SIM system resolution test and super resolution imaging of biological samples. (a) WF image of $100 \mathrm{~nm}$ fluorescent beads. (b) SIM image of the same region as (a). (c) FRC analysis of the resolution using WF and SIM. (d) Image of the NPC of the COS-7 cell in which the left part is the WF result and the right part is the SIM result. (e) WF and SIM images of microtubules of the COS-7 cell. (f) and (g) Magnified view of the yellow area of (e). (h) Normalized intensity profiles along with the marked arrows in (f) and (g). Scale bar: (a) and (b) $0.5 \mu \mathrm{m}$; (d) - (g) $1 \mu \mathrm{m}$.

more reliable when analyzing the resolution because it is a correlation analysis of the whole image at different noise levels, independent of the subjective choice of the experimenter. The red line indicates the conventional result with a resolution of $262.4 \mathrm{~nm}$, which is slightly larger than the theory limit $0.61 \lambda / \mathrm{NA}=250 \mathrm{~nm}$. The blue line indicates the SIM super-resolution result with a resolution of $140.0 \mathrm{~nm}$, which nearly doubles the resolution of the wide-field result. The pattern period we used here in the experiment is $303 \mathrm{~nm}$. The lowest fringe period achievable in the $561 \mathrm{~nm}$ illumination condition is $188 \mathrm{~nm}$.

To further evaluate the applicability of the LiDMD-SIM system for biological sample imaging, we image the NPC and microtubule of COS-7 cells, respectively. The reconstruction is processed using fairSIM. ${ }^{29}$ Here, the NPC was labeled with Alexa Fluor 555 and tubulin was stained with TMR. As shown in Figs. 4(d) and 4(e), the resolution of the SIM reconstructed images is significantly higher than that of the wide-field images. Compared to Figs. 4(f) and 4(g), we can see that two microtubule fibers, which are non-separable in the WF image, could be clearly distinguished in SIM. Meanwhile, we obtained normalized intensity profiles [as shown in Fig. 4(h)] of regions indicated by yellow arrows and red arrows in Figs. 4(f) and 4(g).

Multi-color SIM plays a vital role in studying intracellular organelle interactions. However, the blaze wavelength is already determined in the LiDMD-SIM system. Our first effort to achieve multicolor SIM imaging is to find the wavelengths that blaze at the same incident angle (Fig. S2, supplementary material). Afterward, we take the ON state and the OFF state of the DMD as two different blaze angles, to enable even more imaging colors. Our proposed LiDMDSIM can potentially perform four-color imaging, which matches the performance of the existing SIM system, yet in a very cost-efficient configuration.

In conclusion, a cost-effective laser interference-based DMDSIM system was demonstrated to be feasible in this paper. The effect of intensity uniformity of \pm 1 order diffracted beams on the LiDMDSIM system is illustrated, and we verify the characteristics of DMD diffraction by theory and experiment. Then, the relationship between the blaze condition with the wavelength and the incident angle is summarized as guidance to build a LiDMD-SIM system. Based on the theoretical analysis, we homebuilt a LiDMD-SIM imaging system with an $\sim 2$ lateral resolution improvement. The filament structure of microtubules of COS-7 cells can be clearly distinguished by LiDMD-SIM compared to wide-field microscopy. We further propose a multi-color SIM imaging concept by employing both DMD ON and OFF states at different incident angles to satisfy the blaze condition, which will extend the application of LiDMD-SIM to match the state-of-the-art multicolor SIM systems with a significantly reduced cost.

See the supplementary material for detailed information on (1) the comparison of LED projection-based and laser interference-based DMD-SIM and (2) the simulation and the concept of multi-color imaging.

\section{AUTHORS' CONTRIBUTIONS}

M.L. and Y.L. contributed equally to this work.

This work was financially supported by the Beijing Natural Science Foundation (No. JQ18019), the National Natural Science Foundation of China (Nos. 61729501, 31971376, 51720105015, and 61705252), the National Key Research and Development Program of China (No. 2017YFC0110202), the China Postdoctoral Science Foundation, Innovative Instrumentation Fund of PKU, AustraliaChina Joint Research Centre for Point-of-Care Testing (ACSRF65827, SQ2017YFGH001190), Science and Technology Innovation Commission of Shenzhen (KQTD20170810110913065), and the Natural Science Foundation of Jiangsu Province (No. BK20170388).

\section{DATA AVAILABILITY}

The data that support the findings of this study are available upon reasonable request from the corresponding authors.

\section{REFERENCES}

${ }^{1}$ A. Lal, C. Shan, K. Zhao, W. Liu, X. Huang, W. Zong, L. Chen, and P. Xi, IEEE Trans. Image Process. 27, 4555 (2018).

${ }^{2}$ X. Huang, J. Fan, L. Li, H. Liu, R. Wu, Y. Wu, L. Wei, H. Mao, A. Lal, P. Xi, L. Tang, Y. Zhang, Y. Liu, S. Tan, and L. Chen, Nat. Biotechnol. 36, 451 (2018).

${ }^{3}$ A. Lal, C. Shan, and P. Xi, IEEE J. Sel. Top. Quantum Electron. 22, 50 (2016).

${ }^{4}$ K. Chu, P. J. McMillan, Z. J. Smith, J. Yin, J. Atkins, P. Goodwin, S. Wachsmann-Hogiu, and S. Lane, Opt. Express 22, 8687 (2014).

${ }^{5}$ Y. M. Sigal, R. Zhou, and X. Zhuang, Science 361, 880 (2018).

${ }^{6}$ Y. Liu, Y. Lu, X. Yang, X. Zheng, S. Wen, F. Wang, X. Vidal, J. Zhao, D. Liu, Z. Zhou, C. Ma, J. Zhou, J. A. Piper, P. Xi, and D. Jin, Nature 543, 229 (2017).

${ }^{7}$ X. Yang, K. Zhanghao, H. Wang, Y. Liu, F. Wang, X. Zhang, K. Shi, J. Gao, D. Jin, and P. Xi, ACS Photonics 3, 1611 (2016).

${ }^{8}$ X. Yang, H. Xie, E. Alonas, Y. Liu, X. Chen, P. J. Santangelo, Q. Ren, P. Xi, and D. Jin, Light Sci. Appl. 5, el6134 (2016).

${ }^{9}$ M. Bates, H. Bo, M. J. Rust, G. T. Dempsey, W. Wang, and X. Zhuang, Nat. Methods 3, 793 (2006).

${ }^{10} \mathrm{~B}$. Huang, W. Wang, M. Bates, and X. Zhuang, Science 319, 810 (2008).

${ }^{11}$ K.-H. Song, Y. Zhang, G. Wang, C. Sun, and H. F. Zhang, Optica 6, 709 (2019).

${ }^{12}$ R. Heintzmann and T. Huser, Chem. Rev. 117, 13890-13908 (2017). 
${ }^{13}$ D. Li, L. Shao, B.-C. Chen, X. Zhang, M. Zhang, B. Moses, D. E. Milkie, J. R. Beach, J. A. Hammer, M. Pasham, T. Kirchhausen, M. A. Baird, M. W. Davidson, P. Xu, and E. Betzig, Science 349, aab3500 (2015).

${ }^{14}$ Y. Guo, D. Li, S. Zhang, Y. Yang, J. J. Liu, X. Wang, C. Liu, D. E. Milkie, R. P. Moore, and U. S. Tulu, Cell 175, 1430 (2018).

${ }^{15}$ J. Nixon-Abell, C. J. Obara, A. V. Weigel, D. Li, W. R. Legant, C. S. Xu, H. A. Pasolli, K. Harvey, H. F. Hess, E. Betzig, C. Blackstone, and J. LippincottSchwartz, Science 354, aaf3928 (2016).

${ }^{16}$ K. Zhanghao, X. Chen, W. Liu, M. Li, Y. Liu, Y. Wang, S. Luo, X. Wang, C. Shan, H. Xie, J. Gao, X. Chen, D. Jin, X. Li, Y. Zhang, Q. Dai, and P. Xi, Nat. Commun. 10, 4694 (2019).

${ }^{17}$ B. J. Chang, L. J. Chou, Y. C. Chang, and S. Y. Chiang, Opt. Express 17, 14710 (2009).

${ }^{18} \mathrm{~W}$. Zong, X. Huang, C. Zhang, T. Yuan, L-L Zhu, M. Fan, and L. Chen, Biomed. Opt. Express 5, 1530 (2014).

${ }^{19}$ C. Chong, L. Simin, W. Gang, L. Yong, W. Linbo, Y. Guang, J. Xin, and L. Hui, Biomed. Opt. Express 11, 1203 (2020).

${ }^{20}$ D. Dan, M. Lei, B. Yao, W. Wang, M. Winterhalder, A. Zumbusch, Y. Qi, L. Xia, S. Yan, and Y. Yang, Sci. Rep. 3, 1116 (2013).
${ }^{21}$ D. Dan, B. Yao, and M. Lei, Chin. Sci. Bull. 59, 1291 (2014).

${ }^{22}$ A. Sandmeyer, M. Lachetta, H. Sandmeyer, W. Hübner, T. Huser, and M. Müller, bioRxiv:797670 (2019).

${ }^{23}$ M. Li, Y. Li, W. Liu, K. Zhanghao, and P. Xi, arXiv:1910.13896 (2019).

${ }^{24}$ D. Dudley, W. M. Duncan, and J. Slaughter, "Emerging digital micromirror device (DMD) applications," Proc. SPIE 4985 (2003).

${ }^{25}$ S. Popoff, See http://wavefrontshaping.net/index.php/57-community/tutorials/ spatial-lights-modulators-slms/131-setting-up-a-dmd-diffraction-effects for "[tutorial] Setting up a DMD: Diffraction Effects" (2016).

${ }^{26}$ P. Kner, B. B. Chhun, E. R. Griffis, L. Winoto, and M. G. Gustafsson, Nat Methods 6, 339 (2009).

${ }^{27}$ H. W. Lu-Walther, M. Kielhorn, R. Forster, A. Jost, K. Wicker, and R. Heintzmann, Methods Appl. Fluoresc. 3, 014001 (2015).

${ }^{28}$ R. P. J. Nieuwenhuizen, K. A. Lidke, M. Bates, D. L. Puig, D. Grünwald, S. Stallinga, and B. Rieger, Nat. Methods 10, 557 (2013).

${ }^{29}$ M. Müller, V. Mönkemöller, S. Hennig, W. Hübner, and T. Huser, Nat. Commun. 7, 10980 (2016). 